\title{
The Practical Research on Resources and Achievement Transformations of Traditional Skill Competition in Traditional Chinese Medicine
}

\author{
Yan Zhu \\ Biological Engineering Department. \\ Liaoning economic management cadre Institute \\ Shenyang, China
}

\author{
Jiao Feng \\ Biological Engineering Department. \\ Liaoning economic management cadre Institute \\ Shenyang, China
}

\begin{abstract}
The educational reform in vocational education and its leading role in professional development were enhanced. Combined with our professional advantages, we had analyzed the implementation plan of national traditional skill competition in TCM. We had focused on the research of resources and achievement transformation of skill competition to promote talent-development of Chinese pharmacological specialty. The results could optimize teaching theories and enrich practice teaching resources. It could also improve the evaluation methods of teaching, theory and practical teaching. The promotion and application of results in competition were expanded in teaching processes. Resources and achievement transformations of competition were gained increasing attention.
\end{abstract}

Keywords-Traditional Chinese Medicine (TCM); national traditional skill competition in TCM; resources and achievement transformations; skills training

\section{INTRODUCTION}

Traditional skills of TCM are the quintessence of Chinese culture. It is important in TCM industry all the time. In recent years, sorts of competitions had been organized by municipal and provincial governments in China. The educational reform in vocational education and its leading role in professional development were enhanced. The promotion and application of results in competition were expanded in teaching processes [1]. Resources and achievement transformations of competition were gained increasing attention.

\section{SIGNIFICANCE OF RESOURCES AND ACHIEVEMENT TRANSFORMATIONS OF TRADITIONAL SKILL COMPETITION IN} TCM

The innovative and developing resources and achievement transformations were formed through extracting from items of national traditional skill competition in TCM. The resources and achievements, including training programs, skill points and evaluation indexes, were set according to the task module or skill module. We had formed power points, operating procedures, demo videos, teaching strategies, training guidance and resources of the training base.
Combined with our professional advantages, we had analyzed the implementation plan of national traditional skill competition in TCM. We had focused on the research of resources and achievements transformation of skill competition to promote talent-development of Chinese pharmacological specialty. The results could optimize teaching theories, and enriched practice teaching resources as well [2]. They also improved the methods of evaluation which applied to evaluation of teaching, and also showed the spirited image of school in foreign exchange. Good results were gained when the achievements applied to training of contestants in skill competition in TCM. Sustainable development in Chinese pharmacological specialty was promoted by application and resources and achievements sharing. Characteristic specialty was built. Social benefits were gained for school. It was benefit to inherit traditional skill in TCM. TCM cause was inherited and carried forward. Many competitions were carried out by ministry of education and Chinese vocational technology education. Resources and achievement transformations had not formed by higher vocational college, overall they had not reported in literatures at home and abroad [3]. Competition comes from teaching. Educational reform was promoted through training of competition. But resources and achievement transformations of traditional skill competition were not presented, and not transformed in teaching to promote the development in Chinese pharmacological specialty.

\section{MAIN CONTENT OF RESOURCES AND ACHIEVEMENT TRANSFORMATIONS OF TRADITIONAL SKILL COMPETITION IN TCM}

A. Summary module of traditional skill competition in TCM

This module fully reflected the characteristic of skill examination. It contained basic resources and expanded resources. Skill summary included Chinese drugs macroscopic identification and effect, skills of Chinese traditional medicine dispensing, Chinese herbal medicine processing and authenticity identification. Training programs, skilled points, evaluation indexes were 
also included. The skilled introduction and training program were established according to skills requirements and training procedures of competition. Skilled points and evaluation indexes were established on the basis of competition. It was benefit to summarize skilled training points and skilled evaluation standard.

\section{B. Teaching module of traditional skill competition in TCM}

This module mainly included task module and skill. Power Points, operating procedures and demo videos were produced, which included Chinese drugs macroscopic identification and effect, skills of Chinese traditional medicine dispensing, Chinese herbal medicine processing and authenticity identification.

\section{Expanded resources module of traditional skill competition in TCM}

This module reflected the features of traditional Chinese pharmacological skills, which included expert comments, showing of traditional Chinese pharmacological skills, item bank of each items, material repository, etc. The results of traditional skill competition in TCM were transformed into teaching resources, and they were expanded and applied in teaching [4]. They became the versified ancillary resources to support skilled teaching in order to cultivate talents with good basic theory, practical skill and professionalism.

\section{IMPLEMENTATION OF RESOURCES AND ACHIEVEMENTS TRANSFORMATION OF TRADITIONAL SKILL COMPETITION IN TCM}

Skilled trainings in skill competition were analyzed combined with attending all kind of competitions in recent years. Detailed skilled trainings in every department were summarized which satisfied the demand of knowledge, ability and quality of industrial request [5]. With the increasing demand of skill competition, training methods of each item were explored. During the training process, problems were found and solved. Training and schemes for the examination were quantified. They were also continuously completed to improve training quality. Resources and achievement transformations of traditional skill competition in TCM were consummated by analyzing relevant literature information. The work how to organize the skill competition was perfected. Training work was also. Training effectiveness was increased. Great successes were had by contestants. At the same time, the quality of Chinese herbal medicine would come to a new level [6].

\section{PERSONAL EXPERIENCE OF PARTICIPATING IN TRADITIONAL SKILL COMPETITION IN TCM}

High frequency problems were summarized. The key point of work, main emphasis of setting practical teaching and teaching management in the talent training of higher vocational college were summarized below.

\section{A. Rationalize teaching between theory and practice}

Traditional skill competition in TCM include four items. They are characteristic identification of Chinese drugs (including authenticity identification), macroscopic identification of Chinese drugs, skills of Chinese traditional medicine dispensing and Chinese herbal medicine processing, they were part of main content to spread technique, and also the most practical skill during the process of agriculture, development and utilization in TCM. Students should have a solid theoretical foundation, experienced operation skills and comprehensive ability to use expertise in order to complete all items fastest within the stipulated time. Therefore, we should pay attention to the following aspects in training curriculum.

1) Choose appropriate formal textbook and improve curriculum standards and quality of teaching.

Chinese traditional medicine identification is the compulsory courses of Chinese pharmacological specialty. It introduces identification of medicinal plant, characteristic identification of Chinese drugs, authenticity identification and macroscopic identification. Combined with requirements of competition and actual working condition, characteristic identification of Chinese drugs was carried out generally by experiences such as observing, smelling, touching, tasting, hearing, water test or fire test. Convenient methods of physical and chemical identification were also applied, such as chromogenic reaction, sublimation reaction, fluorescence reaction, etc. As Zongwan Xie said, identify the character of Chinese drugs to discuss the quality of Chinese drugs. It is always the essence of Chinese medicine identification. One item of skill competition requires contestants to identify 40 medicinal herbs. Therefore, correctness of standards was ensured. When curriculums were set, class were distributed properly into practice teaching between characteristic identification and physical and chemical identification in order to strengthen students' handling ability [7]. The ability of authenticity identification was improved to the quality of teaching.

2) Introduce Chinese pharmacopoeia(2015 edition, Volume I) into curriculum.

China Pharmacopoeia (2015 edition, Volume One) contains 618 categories of medicinal materials and cut crude drugs. It is the code of pharmic quality specifications. It is also the evidence of pharmic production, examination supply and use. It guides agriculture, processing, identification and quality standards of TCM to insure pharmaceutical safety and effectiveness. Students could be helped to understand and remember the character of Chinese herbal medicine by introducing Chinese pharmacopoeia (2015 edition, Volume I) into curriculum. It would better to guide practice. It was good evidence that the champion of traditional skill competition in TCM could memorize 618 categories of medicinal materials and cut crude drugs in China Pharmacopoeia (2015 edition, Volume One) well.

3) Introduce Chinese traditional medicine dispensing into practice course, and cultivate excellent dispenser with quality of good accuracy and high-speed.

Chinese drug dispensing should be introduced into independent practice course in the curriculum. Chinese drug dispensing is the necessary professional accomplishment of getting a job. The quality of Chinese drug dispensing directly affected clinical effectiveness. The quality and drug safety was assured in the condition of correctly Chinese 
herbal medicine, exact dosage and disposing of special drugs or valuable drugs properly. Only with great skills of Chinese drug dispensing, we could be prevented and cured. Skills of Chinese drug dispensing focused on students' professional foundation knowledge. Skills of operation were made claims to students. In the competition, students should complete dispensing 10 drugs (without labels) with a small steelyard within the stipulated time. Referees check the results with electronic scale. On the basis of identification of drugs, grabbing drugs exactly one time was a fortress of results. Students with good accuracy and high-speed should be cultivated with accurate dispensing, speedy dispensing, seriously checking, signature and package of special drugs. Students' handling ability could be improved through introducing Chinese traditional medicine dispensing into independent practice curriculum.

4) Curriculum standard of Chinese drug processing was improved

Chinese drug processing is a traditional methods and technology of processing medicinal materials into the cut crude drugs according to the requirement of TCM. It is gems of Chinese culture of TCM inheritance. Traditional processing methods includes processing with water, processing with fire, processing with both water and fire and other processing methods. Stir-frying and stir-frying with liquid were the frequently-used processing methods clinically. According to the demand of skill competition, these two methods should be taken as the key point. Practice should be directed with theories to improve the training of operating skills.

\section{B. Teaching, studying and management was all promoted by skill competition}

\section{1) Teaching was promoted by skill competition.}

Traditional skill competition in TCM had cultivated a group of highly qualified team of double-typed teachers. In order to teach students well, teachers should have intense sense of responsibility, higher professional theory level and strong professional ability. Schoolenterprise cooperation and simulate pharmacy were both promoted through attending skill competition. At the same time, the investment construction dynamics of Chinese drug processing laboratory was increased. Teaching was optimized [8].

\section{2) Studying was promoted by skill competition.}

Good study style of putting skills to work was built in students. Excellent students with comprehensive quality were selected to attend the senior competition by inner school competitions. It had contributed to set clear learning goal for students to practice basic training hardly. Specialized skills were improved.

\section{3) Management was promoted by skill competition.}

Various professional competitions were held in school in order to give students the direction of study. The Afterclass life was enriched and specialized skills were also enhanced [9]. The foundation for getting jobs was created. It is a major systematical design and innovation to establish the system of traditional skill competition in
Chinese education. Vocational educational reform was deepened. Integration of production and education and schoolenterprise cooperation were promoted. A blend of talent development and industrial development was promoted. Vocational education emphasizes taking cultivation of professional skills as fundamental and it meets the demand of subjects, teaching and society. Professional theory is for the degree of necessary and sufficient. So practice of relatively teaching system was built, focusing on the technology application in designing curriculum. Practices in Chinese pharmacological skills were carried out actively. Specialized skills and competitive skills of students were promoted. Technology applied talents and technology operated talents were cultivated. Successors were cultivated for inheritance of Chinese pharmacological characteristic skill.

\section{CONCLUSION}

Professional skills competition as a new thing, its meaning has no event itself, but more on promotion of higher vocational colleges training. A kind of promoting development of "forced" mechanism was formed for higher vocational education talent training by Professional skills competition [10]. Vocational education had promoted the development of persisting with the guide of competition. The nature of vocational education features had leaded returning. Thus the sustainable development of higher vocational education had realized in our country.

Professional skills competition reflected the latest demand for high-skilled talents in the industries and enterprises in every level. The latest development of industries and enterprises for skills required were also reflected. Therefore, according to their own actual situation, the higher vocational colleges should participate in the professional skills competition actively. As an opportunity, higher vocational talents training mode continuously explored and researched. High-skilled talents who conforms to the industries and enterprises practical demand were cultivated. Therefore, the sustainable development of higher vocational education in our country was realized. Practice proves that the players of skills competition training has advantage in the employment competitiveness and the sustainable development ability. They also were popular with employers.

\section{ACKNOWLEDGMENT}

The work was supported by Liaoning education planning project (JG16EB130) and Liaoning Economic Management Cadre Institute (Ljgykt-yb1714).

\section{REFERENCES}

[1] Mingliang Liang, and Huimin Zhang, "National higher vocational students' skills competition on the promotion of teaching reform and talent training," Experimental Technology And Management, vol. 28, pp 161-163, February 2011 (in Chinese) (references).

[2] Yongxin Liu, and Xuesen Du, "The relationship between professional skills competition and higher vocational talents training mode," Education And Vocation, vol. 21, pp. 43-44, July 2014 (in Chinese) (references). 
[3] Wenjuan Liu, Yanli Li, and Zhen Lin, etc., "Influence of professional skills competition on the talent training mode of pharmacy professionals," China Pharmacy, vol. 25, pp. 478-450, February 2014 (in Chinese) (references).

[4] Zhili Wu, "Vocational skills competition countermeasures analysis of skilled personnel training in higher vocational colleges," Communication of Vocational Education, vol. 26, pp. 25-27, 2013 (in Chinese) (references).

[5] Jiaoming Li, "The current situation and thinking of students professional skills competition," Adult Education, vol. 11, pp. 41-42, 2010 (in Chinese) (references).

[6] Zhixian Meng, "Skills competition in vocational education," Journal of Tianjin Vocational Institutes, vol. 13, pp. 3-10, April 2011(in Chinese) (references).
[7] Amin Zhang, and Dazhen Xu," The current situation analysis and countermeasure research of national vocational students skills competition," Higher Vocational Education-Journal Of Tianjin Vocational Institute, vol. 22, pp. 13-15, October 2013 (in Chinese) (references).

[8] Jing Mi, Modern Vocationalism. Tianjin university press, 2010, pp.6873 (in Chinese) (references).

[9] Zhaoxu Li, "The promotion of professional skills competition to vocational education curriculum reform," Journal of Qingyuan Polytechnic, vol. 2, pp. 115-118, 2009 (in Chinese) (references).

[10] Binghe Chen, "Research on the value of skills competition to higher vocational talents training," Chinese Vocational And Technical Education, vol. 9, pp. 36-37, March 2008 (in Chinese) (references). 